\title{
Neuropädiatrische Diagnostik und Rehabilitation bei sehr und extrem Frühgeborenen
}

\author{
Neuropediatric Diagnosis and Rehabilitation of Very and Extremely Premature Infants
}

H. M. Straßburg

Bibliografie

DOI http://dx.doi.org/ $10.1055 / \mathrm{s}-0030-1268416$

Klin Padiatr 2010; 222: 425-426

(c) Georg Thieme Verlag KG

Stuttgart · New York

ISSN 0300-8630

Korrespondenzadresse

Prof. Dr. Hans-Michael

\section{Straßburg}

Universitäts-Kinderklinik

Ärztlicher Leiter des SPZ

„Frühdiagnosezentrum“

Präsident der Deutschen

Gesellschaft für Sozialpädiatrie

und Jugendmedizin (DGSPJ)

Josef-Schneider-Straße 2

97080 Würzburg

Tel.: +49/931/20127709

Fax: $+49 / 931 / 20127858$

strassburg@mail.uni-

wuerzburg.de
Die frühzeitige Erkennung zerebraler Funktionsstörungen und deren Rehabilitation bei sehr und extrem Frühgeborenen ist einerseits ein faszinierendes Thema mit vielen innovativen Ansätzen, andererseits aber auch ernüchternd. Zunehmend müssen die bekannten Komplikationen wie globale Intelligenzminderung, Zerebralparese und zentrale Sehstörung differenziert werden.

In einem Review-Artikel in Lancet Neurology hat J. J. Volpe [12] die strukturellen Schädigungen bei extrem Frühgeborenen „ein komplexes Amalgam von destruktiven und entwicklungsbedingten Störungen“ genannt. So können nicht nur die bekannten Veränderungen der weißen Substanz im Sinne einer periventrikulären Leukomalazie, sondern auch Schädigungen in den Basalganglien, dem Hirnstamm, dem Kleinhirn und der Hirnrinde nachgewiesen werden.

Die Möglichkeiten der hochauflösenden Kernspintomografie mit 3 Tesla werden ausführlich in der Arbeit von M. Born et al. in dieser Ausgabe dargestellt [3]. Neben den seit vielen Jahren bekannten Möglichkeiten der Diagnostik mittels Ultraschall gibt es zunehmend Erfahrungen, in der Initialphase auf der Frühgeborenenintensivstation mit dem amplitudenintegrierten Langzeit-EEG die Hirnfunktionen kontinuierlich zu überwachen, um z.B. genauere Hinweise für Versorgungsstörungen oder zerebrale Anfälle zu erhalten [6].

Zur Früherkennung von motorischen Entwicklungsstörungen haben sich die videogestützten Analysen der Spontanbewegungen (General Movements) bewährt, aber auch entwicklungsneurologische Screeningmethoden wie das Newborn Individualised Developmental Care and Assessment Program (NIDCAP). Auch wird versucht, die General Movements durch automatisierte Bewegungsanalysen besser objektivieren zu können $[5,7,11]$.

Die vom Gemeinsamen Bundesausschuss für Deutschland beschlossene und seit dem 1.4.2009 gültige obligate Untersuchung aller Frühgeborener unter $1500 \mathrm{~g}$ im Alter von korrigiert 2 Jahren kann als erster Schritt für eine standardisierte Entwicklungsdiagnostik angesehen werden, mit der aber bei mindestens einem Drittel der Kinder keine definitive Aussage über die voraussichtliche Langzeitprognose gemacht werden kann [11].

In vielen Arbeiten der vergangenen Jahre konnte eindeutig gezeigt werden, dass die psychosozialen Begleitumstände in der Familie, insbesondere die Ausbildung der Mütter, für die Prognose des zu früh geborenen Kindes von wesentlicher Bedeutung sind. D. Wolke und Mitarbeiter konn- ten darüber hinaus zeigen, dass insgesamt die Prognose von männlichen Frühgeborenen schlechter ist und dass bei einem Gestationsalter unter 32 Schwangerschaftswochen zunehmend biologische Faktoren die Prognose beeinflussen, während bei einem Gestationsalter von mehr als 32 Schwangerschaftswochen soziale Faktoren und frühzeitige Interventionsmaßnahmen wirksamer sind [8]. Aktuell sind von Mitgliedern der Gesellschaft für Neuropädiatrie und der Deutschen Gesellschaft für Sozialpädiatrie Leitlinien zur Nachbetreuung von Frühgeborenen konsentiert worden, die in Kürze von der AWMF publiziert werden.

Von zunehmender Bedeutung für die neuropädiatrische Beurteilung von Frühgeborenen sind sicher differenzierte neuropsychologische Untersuchungen nach dem 5. Lebensjahr, wie sie in der EpiCURE-Studie von Marlow und Wolke [8] bereits ausführlich eingesetzt worden sind. Ob die Verwendung der klassischen Intelligenzteste wie HAWIK-IV und Kaufman-ABC ausreichend ist, um die Ursachen für die Entwicklungsproblematik vor allem der extrem Frühgeborenen genauer zu definieren, ist noch umstritten. Zunehmend gibt es auch Hinweise dafür, dass umgekehrt proportional zum Gestationsalter Verhaltensprobleme wie ADHS, emotionale Störungen und Einschränkungen des Selbstwertgefühls eine Rolle spielen.

Medikamentöse Behandlungen zur Verbesserung der kognitiven Funktionen waren bisher praktisch immer ohne Erfolg - eine Ausnahme bildet die frühe Behandlung mit Erythropoietin bei extrem Frühgeborenen mit intraventrikulären Blutungen, allerdings müssen die Ergebnisse von Neubauer et al. noch in nachfolgenden Studien überprüft werden [9].

Neben den hier angeführten diagnostischen Methoden muss bei den sehr und extrem frühgeborenen Kindern, ihren Eltern und Geschwistern besonders auf eine Stabilisierung der psychosozialen Situation geachtet werden. Dies ist zum einen durch sozialpädiatrische Nachsorgekonzepte, durch die Betreuung mit ambulanten Kindergesundheits- und Krankenpflegefachkräften, vor allem aber durch heilpädagogische Frühförderstellen mit der Möglichkeit des Hausbesuches zu gewährleisten.

Seit vielen Jahrzehnten hat es immer wieder Versuche gegeben, die Entwicklung von HochrisikoFrüh- und Neugeborenen durch möglichst frühzeitige physiotherapeutische Maßnahmen wesentlich zu beeinflussen. Zusammenfassend muss man auf der Grundlage umfangreicher, z.T. auch randomisierter Studien konstatieren, dass mit kei- 
ner spezifischen Methode signifikante Veränderungen in der Bewegungs- und Gesamtentwicklung dieser Kinder festgestellt werden konnten. Auch die seit vielen Jahren praktizierten Anleitungen von Eltern für den Umgang mit ihren Kindern im Sinne des sog. Handlings müssen skeptisch beurteilt werden $[1,2,4]$. Natürlich ist es bei Kindern mit schweren Bewegungs- und Entwicklungsstörungen unverändert wichtig, dass z. B. zur Vermeidung von Kontrakturen regelmäßige Bewegungsübungen stattfinden. Insbesondere die holländische Arbeitsgruppe um M. Hadders-Algra konnte in sehr umfangreichen Studien in den vergangenen Jahren aber überzeugende Hinweise dafür zusammentragen, dass z.B. mit dem Programm COPCA (Coping with and caring for infants with neurological dysfunction - a family centered program) durch konsequente Förderung der Eigenaktivitäten des Kindes signifikante Verbesserungen in dessen Gesamtentwicklung erreicht werden $[2,4]$.

Die Abkehr von aktiv stimulierenden Methoden direkt am Kind und die zunehmende Hinwendung zu Fördermaßnahmen, bei denen keine Berührung mit dem Kind stattfindet, ist sicher für manchen entwicklungsneurologisch engagierten Kinderarzt/ Kinderärztin und für viele Physiotherapeutinnen und Physiotherapeuten eine deutliche und nicht immer einfach zu akzeptierende Konzeptänderung.

Trotz der eindeutigen Hinweise für erhebliche Entwicklungsrisiken vor allem bei Frühgeborenen mit einem Gestationsalter unter 28 Wochen bzw. $1000 \mathrm{~g}$ Geburtsgewicht ist hervorzuheben, dass der weit überwiegende Teil der heute zu früh geborenen Kinder, selbst mit einem Geburtsgewicht unter $500 \mathrm{~g}$, eine zufriedenstellende bis normale Langzeitprognose mit einer guten Lebensqualität haben kann. Vieles spricht auch dafür, dass nicht nur deutlich mehr extrem Frühgeborene als noch vor 20 Jahren überleben, sondern dass parallel dazu auch Anzahl und Schwere der Behinderungen bei den Überlebenden deutlich abgenommen haben $[8,10]$.

Von daher sollten vor allem die Angehörigen von sehr und extrem Frühgeborenen nicht so sehr auf negative Befunde bei frühen Entwicklungstests und apparativen Untersuchungen hingewiesen werden, sondern auf die Fähigkeiten und selbstständigen Aktivitäten des Kindes. Nicht selten ist hierbei auch eine psychotherapeutische Unterstützung gerade der Mütter notwendig. Andererseits dürfen aber vor allem ab dem zweiten Lebensjahr Hinweise für bleibende Entwicklungsstörungen, z. B. eine Zerebralparese oder eine zentrale Amblyopie, nicht negiert werden.
Ohne Zweifel ist das interdisziplinäre Betreuungskonzept für die Frühgeborenen und ihre Familien in Deutschland in den vergangenen Jahren vor allem durch die enge Zusammenarbeit der Perinatalzentren mit den niedergelassenen Kinderärzten, den interdisziplinären Frühförderstellen und den Sozialpädiatrischen Zentren deutlich verbessert worden, vieles kann hier auch in der nächsten Zukunft noch weiter verbessert werden. Dennoch ist vor allem bei den extrem Frühgeborenen die Prognose überwiegend durch Fortschritte des prä-, intra- und postnatalen Managements zu erreichen.

Interessenkonflikt: Die Autoren erklären hiermit, dass kein Interessenskonflikt besteht.

\section{Literatur}

1 Anttila H, Autti-Rämö I, Suoranta J et al. Effectiveness of physical therapy interventions for children with cerebral palsy: a systematic review. BMC Pediatr 2008; 8: 14

2 Blauw-Hospers C, de Graaf-Peters VB, Dirks $T$ et al. Does early intervention in infants at high risk for a developmental motor disorder improve motor and cognitive development? Neuro Sci Bio Behav Rev 2007; 31: 1201-1212

3 Born M, Scheef FL, Boecker $H$ et al. Erfahrungen mit der MRT des Gehirns bei Frühgeborenen - Hinweise auf Schädigung der weißen Substanz in P2- und Diffusionswichtung bei 3 Tesla. Klin Padiatr 2010; 222: 443-448

4 Bower E, Michell D, Burnett $M$ et al. Randomized controlled trial of physiotherapy in 56 children with cerebral palsy followed for 18 months. Dev Med Child Neurol 2001; 43: 4-15

5 Hadders-Algra M. General Movements: A window for early identification of children at high risk for developmental disorders. J Pediatr 2004; 145: 12-18

6 Hellström-Westas L, Klette H, Thorngren-Jerneck $K$ et al. Early prediction of outcome with an aEEG in preterm infants with large intraventricular haemorrhages. Neuropediatrics 2001; 32: 319-324

7 Huppertz-Tessler CJ, Als H, Koch L et al. Frühzeitiges entwicklungsneurologisches Screening frühgeborener Kinder von Geburt an. Klin Padiatr 2009; 221: 450-453

8 Johnson S, Fawke J, Hennessy E et al. Neurodevelopmental disability to 11 years of age in children born before 26 weeks of gestation. Pediatrics 2009; 124: E249-E257

9 Neubauer AP, Voss W, Wachtendorf $M$ et al. Erythropoietin improves neurodevelopmental outcome of extremely preterm infants. Ann Neurol 2010; 67: 657-666

10 Strassburg HM, Leimer S, Platz A et al. Langzeitprognose sehr kleiner Frühgeborener in Deutschland. Klin Padiatr 2008; 220: 60-64

11 Voss B, Neubauer AP, Wachtendorf $M$ et al. Neurodevelopmental outcome in extremely low birth weight infants: what is the minimum age for reliable developmental prognoses? Acta Pediatr 2007; 96: 342-347

12 Volpe JJ. Brain injury premature infants: a complex amalgam of destructive and developmental disturbances. Lancet Neurol 2009; 8: 110-124 